\title{
Space-Time Universe versus Energy Driven Time Arrow Universe: Time-Neutrality Confronted with Fundamental Irreversibility
}

\author{
Helmut Tributsch ${ }^{1,2^{*}}$ \\ ${ }^{1}$ Institute for Physical and Theoretical Chemistry, Free University Berlin, Berlin, Germany \\ ${ }^{2}$ Helmholtz Centre Berlin for Materials and Energy, Berlin, Germany \\ Email: helmut.tributsch@alice.it
}

How to cite this paper: Tributsch, H. (2019) Space-Time Universe versus Energy Driven Time Arrow Universe: Time-Neutrality Confronted with Fundamental Irreversibility. Journal of Modern Physics, 10, 1029-1064. https://doi.org/10.4236/jmp.2019.108068

Received: June 15, 2019

Accepted: July 26, 2019

Published: July 29, 2019

Copyright (อ 2019 by author(s) and Scientific Research Publishing Inc. This work is licensed under the Creative Commons Attribution International License (CC BY 4.0).

http://creativecommons.org/licenses/by/4.0/

\section{c) (i) Open Access}

\begin{abstract}
A dynamic interpretation of quantum phenomena based on an energy driven time arrow requires a combined description of matter and information on matter. This information around matter turned out to be gravitation and the fact that a photon is continuously recycled via this information generates an always constant light velocity. These two phenomena, simple consequences of fundamental irreversibility, have mathematically been imposed on empty space for time-neutral spacetime in General Relativity theory. In an irreversible universe such a four-dimensional spacetime would not anymore be required. Another striking difference is the role of time. Clock-time, used in Relativity Theory and found to be relative, is not associated with a generation of changes, being only a scale for measuring changes, based on selected periodic phenomena. The real time in an irreversible world, action time, is the flow of action, as generated by the principle of least action, or, alternatively, the loss of information on the past. In contrast to clock-time, action time is invariant with respect to relativistic transformation and also facilitates self-organization of matter and information. Gravitation as information on matter with the aim of imposing the principle of least action also provides the link between quantum world and cosmology, which Relativity Theory cannot provide. Relevant aspects of both theoretical approaches, with special emphasis on already experimentally verified spacetime phenomena, are critically analysed. While Relativity Theory, which is relying on time-neutral laws, is applied to support a chaotically exploding Big Bang scenario, the fundamentally irreversible universe subject to an energy driven time arrow is characterized by self-organization of energy, matter and information yielding an intelligent and creative "Self-Image" universe, which is able to periodically regenerate itself. Arguments for a fundamentally irreversible energy driven nature
\end{abstract}

${ }^{\star}$ Retired. 
include, apart from explaining experimental support for Relativity Theory differently, the simple, straightforward derivation from a dynamically interpreted principle of least action, the elimination of quantum and cosmological paradoxes and the more sensitive and flexible information-technology based (digital) nature of gravitation as compared with the analogue "bent space" gravitation.

\section{Keywords}

Time-Neutrality, Irreversibility, General Relativity Theory, Big Bang Universe, Self-Image Universe, Information

\section{Introduction}

\subsection{Why Is It Necessary to Question Space-Time}

When ad-hoc postulates of an established time-neutral theory can readily be derived from a new, simpler irreversible one, a review of the scientific situation concerned is required. This is done with this publication. During a recent effort, aimed at investigating and eliminating paradoxes in physical theories the principle of least action was interpreted in a dynamic way leading to the conclusion that energy should not be considered to be a scalar quantity, only with the ability, not the interest to do work. It should be considered to be a dynamic variable, a vector, with an interest to do work and the ability to drive time [1]. The drive of free energy to do work should be expressed in the property to decrease its presence per state towards a redistribution of energy on many states in form of entropy increase and not anymore available energy. Since energy is conserved, the real changes proceeding during this reaction concern the abandonment and a reduction of information on the way from available to not anymore available energy (equivalent to a low information content). As a result, a dynamic time is obtained, expressed as a flow of action (energy times time) as a consequence of approaching least action. Alternatively, the turnover, the drain of information during energy conversion (the loss of information on the past) can also be defined as time arrow. They are, in both expressions, invariant against transformation to moving systems and represent the "dynamic" time arrow as trace of energy. It makes, in contrast to the presently established paradigm of time-neutrality, nature fundamentally irreversible and time oriented. This had also to be considered in quantum theory with the consequence that particle and wave are not energetically equivalent but that the spread-out wave, with its higher entropy content, has to be restored into a particle with the help of information, set aside for this purpose. As a consequence, a quantum state has to be described in terms of particle or wave including the information on the state of both of them. This information image on matter not only eliminated quantum paradoxes, but also helped to get insight into relevant physical contexts, which remained blurred by irrationality and paradoxes [1] [2]. The argument that a particle-wave duality cannot be considered as given (as assumed in conventional 
quantum theory), but has to be mediated by information can be supported with the example of technical analogue-digital-analogue converters, e.g. applied in cellular phones, which also have to use information for analysing, digitalizing, processing and reconstruction, but also for minimising energy needs required for handling information. Without information two different manifestations of energy, the analogue and the digital signal, could technically not be interconverted. Why has this principle not been considered in the particle-wave duality?

The information on matter, needed to mediate particle-wave duality in an irreversible world, which has an energy content, turned out to be what is called gravitation, and a photon, travelling and using this information for particle-wave interconversion (compare Figure 1, top right), maintains the same properties,

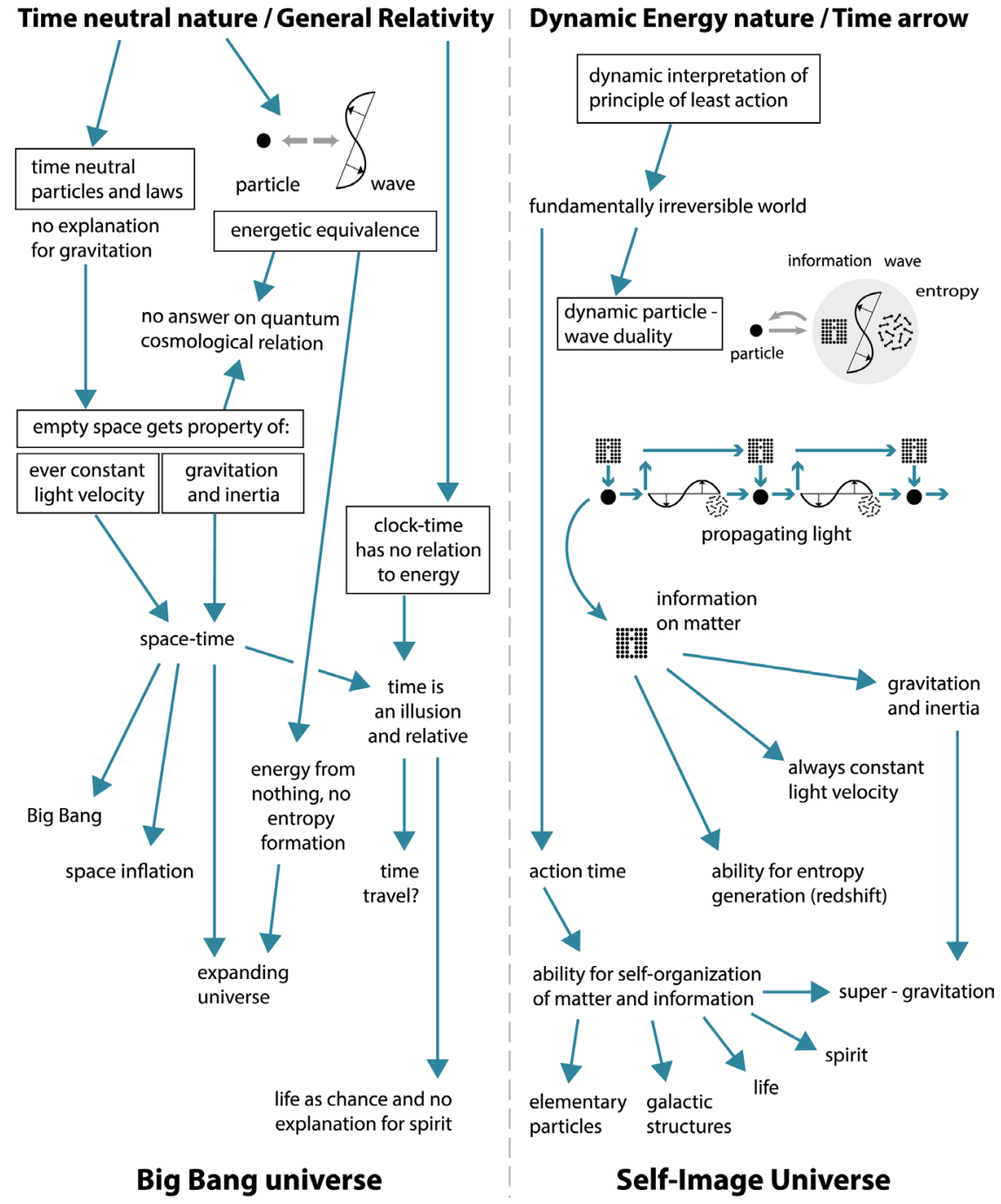

Figure 1. Schemes explaining assumptions (in rectangles) involved in the Time Neutrality paradigm and General Relativity theory (left) as compared with the starting assumptions, derived from the principle of least action, related to the Dynamic Energy (Time Arrow) theory (right). Also simplified explained are the dynamic quantum state with the mediating information and propagating light with the intermediate form as information (visualized as dotted square expressing “i”). Consequences and explanations are also included. 
including its always constant velocity, in relative reference systems. It is like transmitting digital information to a flying airplane. The information received is independent of flight direction and flight velocity. Summarizing, the irreversible world, subject to the energy driven time arrow, yields the always constant, absolute light velocity and gravitation (including inertia within a situation of equivalence, when the drive to reduce the presence of energy per state is violated) as fundamental properties deducible from dynamic quantum states.

This is a very stimulating result, however also highly intriguing, since the established and experimentally well tested Relativity Theories were, one century ago, developed for the purpose of explaining exactly these two phenomena. It is well known that after efforts with an ether theory failed, Einstein simply stated that these experimentally verified phenomena of the always constant light velocity and of gravitation (including inertia) are imposed by empty space, claiming space-time properties. The necessary field equations for space were developed and adapted accordingly. Now, after one century of discussions and experimentation the Relativity Theory is so well accepted that criticism is considered not anymore relevant. The four-dimensional spacetime, as well as various relativity phenomena and ideas, including time dilation and time travel, the Big Bang scenario, space inflation, universe expansion, black holes and gravity waves are already discussed even in schools as part of the now established space-time concept of our universe [3].

The situation encountered is quite remarkable: On one hand there is the fully developed science structure based on time-neutral concepts with clock-time only used as a scale for measuring changes, and with experimentally well confirmed theories with significant paradoxes and irrational explanations (energy from nothing, effect without cause, inflation of empty space, non-locality, zero point energy, additional dimensions, multi-worlds). On the other hand there is a starting effort [1] [2] [4] [5] in considering nature as fundamentally irreversible, attributing to energy time driving properties and learning how to describe the universe as a fully rationally functioning system.

The time-neutral world concept sees absolute light velocity, gravitation and inertia as properties of empty space. The long searched for link between quantum world and cosmos could still not be identified. Clock-time, with its function as scale for measuring changes and with its origin from periodic phenomena such as pendulum movements, quartz oscillations and electronic transition frequencies in atoms, turned out to be an illusion (opinion also expressed by Einstein).

The Dynamic Energy concept (in the following also named Time Arrow concept), in contrast, sees absolute light velocity and gravitation as local particle properties reflecting mechanisms of information on matter, the information engaged in dynamic particle-wave duality [1]. The connection between quantum world and cosmos is thus immediately given. Time is the flow of action or information lost about the past, invariant upon transformation. 
A fundamental difference between the two models is also that in the Dynamic Energy approach the two properties, absolute light velocity and gravitation, are just side results of quantum reasoning, while, in the Time-Neutrality world model they are, via Relativity Theory, additionally postulated to be properties of empty space. They are postulated as additional phenomena.

As a basis for discussion Figure 1 compares the basic assumptions of the Time-Neutrality paradigm including General Relativity theory (left) with those of the Dynamic Energy concept (right). On the left side it is shown, marked with rectangles, how the (postulated) time-neutral world implemented a (postulated) space-time concept and an (adopted) futile, relative clock time to describe a chaotically exploding universe (here considered necessary, since entropy formation by expanding, propagating photons cannot be adequately considered).

On the right side it is explained, how the Dynamic Energy approach derives irreversibility from the principle of least action and imposes a dynamic particle wave duality mediated by information on matter (marked with dotted squares, visualizing an "i" indicating information). This, together with the notion, equally deducible form the principle of least action, that energy driven time is the flow of action, or the loss of information on the past, is all what is needed to deduce the always constant light velocity, gravitation, entropy formation by spreading, propagating photons, and an entirely different, information dominated universe. The Dynamic Energy approach claims to be able to eliminate quantum paradoxes (effect without cause, non-locality, fundamental uncertainty, zero-point energy) and paradoxes in cosmology (energy from nothing, space inflation, dark matter and energy) [1] [2] [6].

From this comparison it can be deduced, that the two approaches are not compatible in their dealing with always constant light velocity and gravitation (including inertia) and time. The Dynamic Energy model derives its basic claim that energy is fundamentally dynamic and oriented from the principle of least action [1]. The flow of action turns out to be the real time, action time (see below) responsible also for the self-organizing creativity of life and the universe, since feedback processes are facilitated. When applied to quantum processes, dynamic energy requires consideration also of the role of space for energy. It reduces its presence per state, its information content, with respect to both, time and space, while conserving the energy. This is the reason why a particle adopts the form of a wave. In this spread-out form its ability to do work is decreased due to entropy formation (compare particle-wave duality expressed in symbols in Figure 1, top right). The consequence is the need to introduce an information on matter, which is mediating the reversibility of the particle-wave duality. Besides of these reasonable considerations no further assumptions were needed. The meaning of gravitation, the explanation of the ever-constant light velocity, the link between quantum physics and cosmology and the reason for the structural creativity and function of self-organized systems turned out to be logical consequences. In addition, the interpretation of gravitation as information, to- 
gether with the possibility of self-organisation of information, of information on matter, opened the way towards an explanation of super-gravitation in space. Self-organization of information also opens the way towards a more intelligent handling of genetic information and of information of neuronal origin in the brain. It opens the way towards the explanation of mind and spirit.

In contrast, on the basis of the Time-Neutrality paradigm and the General Relativity theory significant claims had to be made in relation to space (Figure 1, left). Space is imagined such that it imposes the always constant light velocity and accelerates matter in such a way that it generates gravitation and inertia. It cannot provide a link to quantum physics, involves irrational mechanisms, daring theoretical interpretations, and cannot explain dark matter (super-gravitation).

However, due to its one century long history and many experimental efforts, there is at presence overwhelming support for Relativity Theory. This is, last not least also due to very costly experiments, which have produced quite tiny measured values, interpreted in favour of Relativity Theory (the LIGO and the Gravity $B$ probe experiments alone have together cost two billion dollars).

What are the prospects of challenging General Relativity under such conditions? Comparing General Relativity theory with the new Dynamic Energy approach may sharpen our understanding of the universe, especially since it is essentially a confrontation of a time-reversible nature with a fundamentally irreversible one. Is nature fundamentally time-neutral even though everything is moving into one direction only and far from equilibrium processes are so dominating in shaping galactic structures and living organisms? The challenge of confronting both theories is also justified because the Dynamic Energy approach is claiming to eliminate the increasing number of paradoxes and irrationalities, which the paradigm of Time-Neutrality and Space-Time has generated. In addition, only one of the two approaches to describe nature can be correct.

In the following it will be attempted to compare and evaluate essential features of the two theories to understand the crucial differences and to identify experimental and theoretical steps for answering the questions posed. While the Dynamic Energy (Time Arrow) approach pictures a highly intelligent, spiritual universe, the Big Bang universe, explained by General Relativity theory, describes a quite primitive, exploding universe, in which life developed by chance without aim, and mind and spirit get no explanation.

The energy driven time arrow approach is still in its infancy and lacks an elaborate mathematical framework. However, it offers explanations for gravitation and the absolute light velocity, which were not artificially attributed to space (as in General Relativity theory), but simply followed from the quest for a description of nature on the basis of an irreversible, energy driven Time Arrow. It also claims, that clock time is just a time scale for measurement, but not representing the real time flow, which is generated by a dynamic energy. The quite dramatic turning-point generated by postulating a fundamentally irreversible world (Figure 1, right) is shown with the following example: Time-neutral energy and 
clock-time enter Heisenberg's uncertainty relation with its controversial consequence for zero-point energy, which turned out to be a relevant point for speculations on evolution of the universe. For a fundamentally irreversible world with a dynamic energy and an energy driven time an entirely different interpretation is unavoidable even for such a very established relation. The energy driven time arrow supports a much more intelligent and creative universe, which can logically be understood and has the potential for renewal and perpetuation via information on matter (see later).

\subsection{History of Criticism and Theoretical Background of Space-Time}

Einstein's two Theories of Relativity find so much support that critical analyses are usually no longer accepted for publication. Is this persuasiveness of the theories based on irrevocable theoretical and experimental facts? Einstein himself once commented to a journalist that it is the "mystery of not understanding that attracts many people who indeed do not understand" [7]. Some understood physics and mathematics, but still could not accept the Relativity Theory. One example is the famous French mathematician Henri Poincaré. Until his death around 1912 he opposed the theory, as Einstein himself reported [7]. Another famous scientist, Paul Ehrenfest, an Austrian professor who taught in Amsterdam, committed suicide in 1933. In a letter to his colleagues, including Einstein, he commented that he could not continue to teach a science that he could no longer follow. Even Albert A. Michelson, who for the first time, long before Einstein took it into account in his theory, demonstrated the amazing constancy of the speed of light, was by no means pleased with the Relativity Theory. He said he would prefer to believe that his measurements were wrong before he believed this theory (quoted in [8]). Other famous scientists who witnessed the rise of relativity and did not accept it were, for example, Ernest Rutherford, Robert A. Millikan, Ernst Mach, Wilhelm C. Röntgen and Nicola Tesla. During the further 20th century and until today many scientists have wondered about and criticized the Theory of Relativity. The "Worldwide List of Dissident Scientists" compiled by Jean de Climont gives many examples [9]. The theoretical physicist and then vice-director at the Space Research Institute of the Russian Academy of Sciences, S. N. Arteha proceeded very thoroughly with his analysis of problems. He produced a book with the title "Criticism of the Foundations of the Relativity Theory" [10]. In it he physically and mathematically investigates every conceivable aspect of both theories of relativity, including their experimental verification, and finds serious contradictions and inconsistencies. He advises a return to the classical idea of space and time.

It is not the subject of the present paper to deal with questions related to the mathematical formalism of Relativity Theory. It should only be mentioned that it is conspicuous, that conservation of energy, momentum and angular momentum are not considered in it, even though far reaching conclusions are drawn on highly dynamic energetic phenomena in the universe (Big Bang, inflation of 
space, Black Holes, expansion of the universe). There is also no explanation on how empty space, without matter and structure in it, can physically develop such sophisticated properties as space-time theory claims (e.g. acceleration of objects, manipulation of time, adjustment of light velocity). Space-time, which will never reveal the physical origin of its properties is, in this respect, a dead end for scientific understanding.

Why is it, after one century of continuous rise and confirmation of Relativity Theory and much unsuccessful criticism now justified to challenge this theory again? There is a significant reason: One is not any more just dealing with a mere criticism, like in numerous earlier efforts. It is for the first time that a counter theory on the basis of much simpler and more reasonable assumptions, which explain the always constant light velocity, gravitation, inertia and time differently, takes shape. It also naturally explains the relation between the quantum world and the cosmos, which Relativity Theory and Standard Model of elementary particles could not provide. This new, alternative universe turns also out to behave much more intelligent than deducible on the basis of the Big Bang scenario. It can explain the thrust of biological evolution as well as evolution of spirit through self-organization of information and replaces the Big Bag explosion of energy from nothing, inflation of empty space as well as accelerating expansion of the universe with a more logic interpretation. In addition, the challenge of questioning Relativity Theory means to simultaneously discuss, whether nature is fundamentally time-neutral, as presently assumed in physics, or fundamentally irreversible, as the Dynamic Energy theory claims. Is our universe governed by fundamentally time-neutral laws and mechanisms, and is time an illusion, even though everything is visibly moving in one direction only? This alone already gives justification for this attempt to question the time neutral world of space-time.

\section{Results}

\subsection{Time Neutrality against Fundamental Irreversibility}

It is well known that present concepts of nature including elementary particle (Standard Model) theory, quantum theory and relativity theory are based on time-neutral concepts. All mechanisms can proceed in positive as well as negative time direction and fundamental laws of physics allow that. The only time orientation presently accepted in physics is that in direction of increasing probability and increasing entropy. A system assumes a more disordered condition characterized by a minimum information on it.

The author, in his effort to demonstrate fundamental irreversibility, has criticised such a concept and its mathematical basis [6] [11]. During mathematical derivation, within the $\mathrm{H}$-theorem, of the entropic time, information on time-neutral particles is reorganized, simplified and partially abandoned. This is done by replacing the initially very exact description by an estimation (Marcovian mixture), a statistical procedure, aimed at predicting the future on the basis of re- 
duced information. Since information has an energy content $(1$ bit $=k T \ln 2)$ energy is thereby thrown away. This explains, why the system assumes directionality and cannot be reverses. There is an additional argument against the entropic time arrow. The system concerned approaches a situation of maximum disorder or minimum information content moving with the entropy content from $S_{1}$ to $S_{2}$ by $\Delta S$. It loses thereby information. Since information has an energy content, where does this energy go? Energy has to be conserved. The concept of a purely entropic time arrow does not work. The author argues that the entropy increase by $\Delta \mathrm{S}$ can be multiplied by the absolute temperature $\mathrm{T}$ to yield the energy quantity $\mathrm{T} \Delta \mathrm{S}$, "entropic" energy. Now, considering the first law of thermodynamics on energy conservation one can ask, where this entropic, not anymore available energy came from. It could only have been derived from Gibbs free energy $\Delta \mathrm{G}$. This however means that not the statistical drive towards disorder, but that a "dynamic" (free) energy as a dynamic variable is the real source of changes towards increasing entropy. Energy has an interest in doing work! This is, of course, not consistent with the presently established concept of a "scalar" energy as a quantity of state, with the ability, but no interest to do work.

When the author studied the important principle of least action he found that also energy within this principle has to be considered "dynamic", because only that way extremal, least action values can be reached at all, and that the principle is expressing a fundamentally irreversible world [1]. The proposed definition of this "dynamic" energy was that it "decreases and minimises its presence per state" thus generating, chaotic, not any more useful energy. This is equivalent to a decrease and minimization of information. Such properties define a fundamentally dynamic time arrow with all its different consequences for explaining the universe.

\subsection{What Is Time in Reality?}

Since antiquity many thoughts have been reported on the meaning of time, and during the last century numerous books have been written on the subject (e.g. [12] [13] [14] [15]). The impression is that no final conclusion has yet been reached. The author has also contributed to the search for the meaning of time [11] leading to the view explained here.

For present science, with its time-neutral particles and laws, and emphasized by personalities like Einstein, time is an illusion. It is just used as an ordering parameter to monitor changes. The Theory of Relativity shows, that time depends on relative movement and each system has its own time. The time used is clock-time, which is just a sequence of numbers, a scale or ruler for measuring changes, without any relation to matter or energy. It can therefore not be directly measured, but has to be derived from energy converting clocks. These clocks, however, do nothing more than to activate a periodical process, such as a pendulum movement, the oscillation of a quartz platelet, or the electron relaxation in an atom. Such time lapses during oscillations, which are just determined by 
natural or material constants and have no relation to energy turnover themselves, are summed up and calibrated against periodic astronomical phenomena to yield seconds, hours and days (compare Figure 2(a)).

It is this clock time, which is just a scale and has nothing to do with energetic processes, which is multiplied with light velocity and became the axis of the fourth dimension in the four-dimensional space-time of Relativity Theory. It is this clock time, which, in the Theory of Relativity is relativistic dilated when calculated for a fast moving reference system. For an atomic clock this means that atomic parameters are thereby changed. This actually happens. Atomic clocks travelling around the globe show time dilations in the order of fractions of a microsecond (e.g. Hafele-Keating experiment). Within the Theory of Relativity part of this effect is attributed to gravitational effects, part to relative movements. But, in fact, only the properties of a scale have been changed due to changed physical parameters. Within the Dynamic Energy concept, it is not the real time which changed but only the scale for measuring it.

Within the concept of a "dynamically" understood energy, and with such a dynamic energy acting via the principle of least action, a real, irreversible energy driven time can readily be defined. It is the flow of action (energy times time), which is activated as the consequence of the principle of least action and can be called action time (Figure 2(b)). It is, for example, the tickling of sand in an hourglass or a stone rolling down a hill and describable as a flow of action. This is, more or less, what the Greek naturalist and philosopher Aristotle, who lived

a)

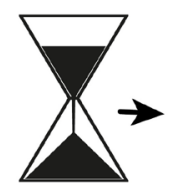

hourglass, symbol for energy-driven action

action

(clock) periodic phenomenon activated in clock

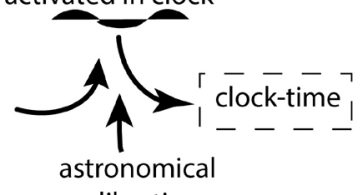

calibration

b)

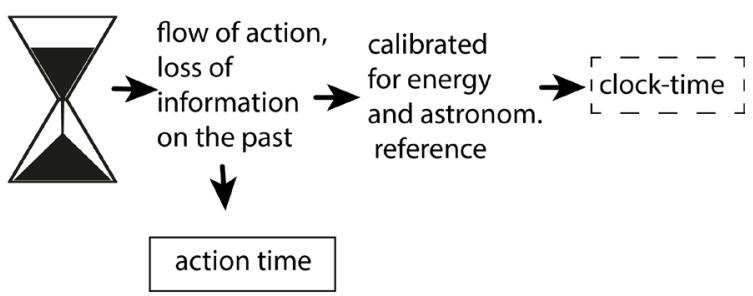

Figure 2. Scheme explaining the difference between clock time (a), which is just a scale for measuring change, and action time, which is here considered to be the real, energy-driven time (b). The symbols on the left side of depictions show a simplified hourglass representing energy turnover and a flow of action, the flowing sand, on the way of fulfilling the least action condition. In an ordinary clock action is just used to activate a calibrated oscillating mechanism, which provides a scale for measuring change. The real time flow is action time, the flow of action as a consequence of energy conversion, or the loss of information (supplied by free energy) on the past. If normalized for energy, clock-time can also be deduced (b). 
in the 4th century BC, observed. He said: "Time is the measure of a movement that takes place from a before to an after". Movement requires energy. Then time is a measure for energy turnover. This is exactly what the Dynamic Energy approach states and it can give additional information: Since dynamic processes are proceeding via a reduction of energy per state this action time equally means "loss of information on the past". Such a statement is comprehensible and fully logical: the information, which we have recognized just instances before in our environment, is gone. Just some fragments of memory remain in our brain.

Why is sand moving in an hourglass? It is moving, because energy (gravitational energy) is dynamically active. What is this activity like, when energy, during energy conversion, remains fully conserved? The above given definition, that "it decreases and minimizes its presence per state" implies that order, available within the energy system is being reduced. This way energy is redistributed. In the case of chemical energy (e.g. a carbon-hydrogen bond), or of a photon as primary energy source, elaborate arrangements of energy and materials are abandoned during the energy conversion process to finally only show low temperature kinetic energy. This shows, that it is order, information about the energy system, which is given up during the energy conversion process to yield disorder while energy in total is conserved. The flow of action (energy times time) in such an energy conversion process $(\mathrm{d}(\Delta E t) / \mathrm{d} t)$ is thus generated by a reduction of order (information) within the energy system. It is consequently equivalent to say that it is the flow of abandoned information on the past $\left(\mathrm{d} I_{a b} / \mathrm{d} t\right)$ that characterizes fundamental energy driven time. In this case, since the lost information concerned is linked to energy, there is no problem with energy conservation. It is considered in the energy balance of the entire process. It is this abandonment of information (on energy) that implements the redistribution and conversion of energy thus causing the flow of action.

Energy driven time, or action time, can therefore be formally written in the following way (here $t$ is the clock time and $\Delta E$ the energy turned over:

$$
\text { energy driven time }=\text { action time }=\frac{\mathrm{d}(\Delta E t)}{\mathrm{d} t}=\frac{\mathrm{d} I_{a b}}{\mathrm{~d} t}
$$

and from this equation clock-time can be deduced

$$
\text { clock time } t=\frac{I_{a b}}{\Delta E}
$$

This clock-time does not any more correspond to the flow of abandoned information due to energy turnover. It is an energy neutral statement of abandoned information per energy turned over. Clock-time is a standard, a calibrated scale for measuring changes. It is for this reason, that clock time is subject to dilation, when transformed within Relativity Theory, since it refers to the energy of moving systems, where energy can be determined to be correspondingly larger.

Both equivalent definitions of the proposed real time arrow (1), the flow of action, and the flow of abandoned information on the past are invariant with re- 
spect to relativistic transformation and can directly be measured, because they are characterized by an energy content. But clock time (2) is not, since the invariant information flux is considered per energy turned over. It is just a scale for measuring time. A certain amount of information turnover per energy is considered and counted as a scale. Relativity theory uses clock time for constructing the fourth dimension and draws important conclusions from relativistic time properties. On the basis of the here given definition of energy driven time, action time (1), it should be this time, action time, and not clock-time (2), which should be used in formulas, which aim at the description of the universe, when highly dynamic mechanisms are to be derived as conclusions.

Figure 2 explains in a simple scheme the difference between clock time (above) and action time (below). In the first case (a) a flow of action is only used to activate periodic phenomena for measuring changes via the clock time. In the second case (b) the flow of action itself is considered to be the energy driven time flow. By dividing it through the turned over energy, clock-time can be obtained also in this case as calibrated scale for measuring change.

\subsection{What Means Gravitation and Always Constant Light Velocity for Space-Time}

Relativity theory implements, via the field equations, relevant experimentally verified properties into empty space: they concern the ability of always sustaining the absolute light velocity and the capacity to simulate gravitation and inertia, while respecting the equivalence principle, by adequately accelerating masses. Since these introduced properties actually prevail, this may explain the astonishing apparent experimental meaningfulness of General Relativity theory. The properties of space yield what has been introduced as a theory. But it is well known that these introduced properties cause the now four-dimensional space-time to bend, since gravity has become a geometric property of space-time. A satellite around a celestial body thereby feels a force at close distance, since it is moving along a curved space tracing its trajectory. For understanding, what it means in practice, when a body is deviated by a bent space around a mass let us look at an example. The difference in gravitation forces, and the degree of bent space, between two neutrons and two weights of one kilo is of the order of $10^{54}$. Gravitation means bending of space and bending is induced by the energy momentum tensor in relativity theory. Can one imagine a detectable bending of space, equivalent to gravitational acceleration, around spherical objects differing by a factor of the order of $10^{54}$ ? A passing and interacting particle should nevertheless be able to register the differences and to respond properly to a highly varying gravitation. A bent space around a mass must communicate itself as an analogue signal. Technical experience shows that an analogue signal (which continuously varies as quantity to be registered) can only be measured within $0.01 \%$ of its maximum signal (three digits behind the comma), and has to be regularly calibrated. Gravitational changes of up to and trespassing a range of $10^{54}$ can never be registered via an analogue signal of bent space around a sphere. How accurate 
are gravitation signals measured in practice? This question will be discussed later.

Within the Dynamic Energy theory gravitation is information with the requirement to reduce energy per state while imposing the principle of least action. Phenomena controlled by information are not limited by analogue restrictions and also readily explain, what happens, when the mentioned requirement is violated and the energy per state tends to increase. If this minimisation condition is violated and energy per state increases, then a counter force results, equivalent to energy per distance travelled. This is inertia fulfilling the equivalence principle. It responds to the gravitation of matter from the entire universe, as Ernst Mach proposed. The Dynamic Energy theory has no problem explaining inertia.

Another question is to understand the implementation of absolute light velocity within the four-dimensional space time. Let us imagine a photon approaching an object coming closer at very high speed. When hitting it light velocity measured on the object must be the known absolute value. When, before the encounter, and how is such an adjustment made in a time-neutral world? It formally works, of course, because mathematics imposes it, but it is not easily understandable. This also concerns the time around masses in space-time. Within the Theory of Relativity time is actually variable and manipulated depending on the distance of a mass. How can, on a physical basis time be manipulated? In terms of an atomic clock this means, as explained before, that atomic parameters must change. They can change due to a changed gravitation, but this does not mean that time itself, the energy driven time, is dilated that way.

When the concept of an energy driven time arrow was applied to quantum phenomena, it turned out that matter (energy), concentrated in a particle, and energy spread out as wave had to be linked via an information image of matter (energy). It has to be set aside to support the back conversion of the wave into the particle [1] [6]. This information around matter has an energy content and was identified as gravitation. Propagating photons, changing from the wave form into the particle form via information imply information-controlled photon properties (compare propagating photon expressed in symbols in Figure 1, top right). Photons are continuously reassembled via information (represented in form of dotted squares), independent of relative movements of the receiver. This works similar as programs for television or information for a $3 \mathrm{D}$ printer are registered on an airplane [2]. They function independent of flight velocity and flight direction. The program for a 3D printer can be used to produce a toy car which travels at an always constant speed. In fact, as a side product of applying the energy driven time arrow to quantum states new explanations both for gravitation and the always constant absolute light velocity in free space were found. Surprisingly, gravitation turned out to be information on matter and the absolute light velocity simply the consequence of involvement of information in the quantum process. The just mentioned problems in understanding gravitation or the always constant light velocity in terms of the space-time concept simply dis- 
appear with the information nature of gravitation. Information can work like that and no postulation needs to be made on highly elaborate properties of empty space as General Relativity theory does it.

This, of course, motivated and urged to explore, whether this supports a fundamentally irreversible universe subject to an energy driven time arrow. It became necessary to confront it with the time-neutral, already well-established universe shaped by General Relativity (Figure 1).

Such a confrontation is, in fact, unavoidable, because only one of these two world models for explaining nature can survive. Absolute light velocity and gravitation are either information-controlled properties of an energy driven time arrow, or they are properties of free space, as General Relativity implemented them. The first approach is entirely rational and quite simple, the second a mathematical construction which generates numerous paradoxes and irrationalities (four dimensions, relativistic time and length changes, time travel, space inflation) which, however, already, according to specialists, has produced surprising experimental support.

\subsection{Relation between Quantum World and Universe}

Since the rise of quantum and Relativity Theory scientists have searched for a unifying link between them. The discipline of quantum gravitation, for example, studies that, aiming for a "Theory of Everything". String-theory is another research orientation, which searches for such a connection. Up to now it was not found.

The energy driven Time Arrow approach, in dealing with quantum phenomena, found this link quite naturally. The self-image of matter in form of information, mediating the particle wave exchange was identified with gravitation and the same information (or gravitation) also controls the dynamics of the universe. A remarkable consequence of this finding is, that what we call gravitation is in fact information on matter. This implies that our universe is essentially controlled by information, which has significant further consequences (see later). But it also readily explains, why the measured difference in gravitation between two neutrons and two weights of one kilo of $10^{54}$ does not pose problems for function and detection. It is a difference in numbers, registered as information without the need of an intermediate registration as analogue signal of space bending. Of course, a big task for the future will be to decipher the information code of nature and to understand, how information can be turned over during generation of action.

\subsection{To What Extent Can Relativistic Phenomena Be Understood Differently?}

Special and General Theory of Relativity have puzzled with their very characteristic phenomena. Below, with relation (3), it is shown how the length of an object is reduced, when its velocity $v$ is approaching the speed of light $c$. It is seen, that it is shrinking and finally disappearing. The next formula (4), also well known, 
is showing, how the time interval $\Delta t_{0}$ between two subsequent instances (e.g. seconds) is increasing with increasing velocity $\mathrm{v}$ of an object. Approaching the light velocity the clock cycle is getting larger and larger until finally clock-time stops. The first example of a rocket, shrinking at high speed, rises the question, how this could occur with a stiff object, which subsequently may land in full size. The second example of time dilation, in turn, is the basis of numerous paradoxes which deal with time travel.

When trying to judge these well known predicted phenomena it is of interest to point out, that these relativistic phenomena are only seen, when the object is analysed in direction of movement. Observers analysing it perpendicular to the movement will not see this effect (Figure 3).

Since objects with simultaneously different spatial measures and different time cannot exist, one is apparently dealing with a problem of measurement. The measurement occurs with light, which serves for transmitting the signals. Two measurements have to be made for measuring length and a time interval respectively. During that interval the object is moving with the velocity $v$. It is learned how the ratio of object velocity $\mathrm{v}$ and light velocity $\mathrm{c}$ is affecting measured data. This is definitively a measurement artefact due to the limited light velocity and not information on the studied object. Indeed, when the light velocity in these formula (3) and (4) is set to become infinite, the relativistic effects just disappear.

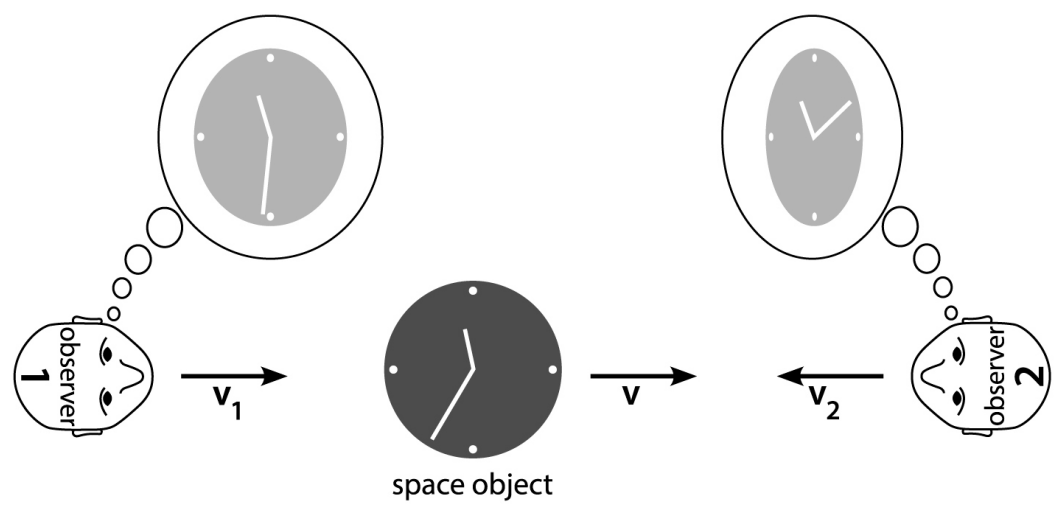

observer 1: slight shrinkage observed observer 2: great shrinkage observed observer 3: no change observed

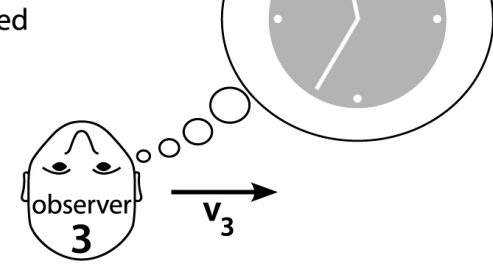

Figure 3. Scheme visualizing a paradox of relativity theory. Three observers with three relative velocities will, according to relations (3) and (4), see the spacecraft shrinking differently and recognize different "time flows" on it. In the direction of the spacecraft's motion, the spacecraft will shrink and there will be a time dilation, different for observers at different speeds. No length or time dilation will be observed perpendicular to the movement. What is the actual length of the spacecraft, what time does it run? 
This means that, in this case of simultaneity, the scale for measuring changes is not compressed or stretched due to a finite transmitting light velocity.

$$
\begin{aligned}
& l=l_{0} \sqrt{1-\frac{v^{2}}{c^{2}}} \\
& \Delta t=\frac{\Delta t_{0}}{\sqrt{1-\frac{v^{2}}{c^{2}}}}
\end{aligned}
$$

The energy driven Time Arrow approach states, that energy converting systems do not follow clock-time (2), but are subject to action time (1). Within the energy driven Time Arrow universe one would not transform the clock-time which is just a ruler or scale for passive measurements of changes. One would transform the real time (1), the flow of action or the flow of abandoned information on the past. Both are relativistic invariant so that moving energy converting objects would see the same time flow. All paradoxes with time travelling then simply disappear.

If one would like to find out clock-time on a moving object, the travellers would have to determine action time, measured for their object, and divide it by the locally turned over energy (2). When the conditions and calibration procedures are the same, also clock-time would be the same. This does not support the statement that every relatively moving object has its own time and that time is an illusion (comment also by Einstein). There is a simple intellectual consideration, that could support such a conclusion. The presently as one of the most distant recognized galaxies, Abell 1835 IR 1916, has a redshift, which indicates it is drifting away with $97 \%$ of light velocity. Clocks there on a similar planet, calculated via relativistic theory, should proceed 4 times slower. Observers there would, however, conclude the same from our galaxy. Does this make sense? Do we have a slowed down evolution, because we see galaxies escaping at a high speed?

The energy driven Time Arrow approach would not expect any difference in time flow when similar environmental conditions prevail. And it would also challenge the claimed high relative velocity seen in the (cosmological) redshift. It is not caused by the expansion of space, but is a consequence of entropy loss by propagating photons (see later).

\subsection{Spacetime Critically Seen in Terms of the Energy Driven Time Arrow}

A significant problem with General Relativity theory, according to the author, is, that the clock-time used is not relevant for transmitting useful information on changes and for calculating action. It is a scale for change only, a sequence of numbers. Another one is that matter and laws which control it were defined to be time neutral. Nevertheless, General Relativity theory is used to justify and describe highly dynamic phenomena, the Big Bang scenario, the inflation of space, to understand Black Holes and to investigate the accelerating expansion of the 
universe.

Today the dynamics of the universe is deduced by back and forward calculating the field equations of General Relativity, which describe the relation between the geometry of space time and the energy-momentum distributed in it. Considering the apparent dynamics of the redshift of galaxies and stars they attribute to the universe an age of 13.8 billion years while it was stretched to a dimension of approximately 78 billion light years. That this rate of expansion exceeded light velocity is not considered a problem, since it is the empty space, which is assumed to have supported this stretching activity. But how can empty space, with no material properties defining it, do this?

The energy driven Time Arrow approach identified time flow as flow of action with changes in the environment (1). It is not just a scale for monitoring changes (2) (compare Figure 2). In addition, gravitation was identified to be information on matter with its root in quantum phenomena. Inertia is just the counter reaction to the fundamental law of Dynamic Energy aiming at reducing the presence of energy per state against a violation of this law. Gravitation and inertia are here not a property of empty space, but information mechanisms implemented on quantum level and, via this information, gravitation, widely present in the universe. The same is true for the interpretation of absolute light velocity. It is simply a property of information handling within the photon and not a property of space either. When these three properties, which, via Relativity Theory have been mathematically and ad hoc implemented into space, are explained in this different way, there is theoretically no basis left for a spacetime universe. However, how can one deal with the significant experimental evidence claimed in support of General Relativity theory and space-time?

With imposed conditions of absolute light velocity and a gravitation subject to the equivalence principle both theories, the General Relativity theory and the Dynamic Energy approach, should have the ability to explain at least part of existing phenomena. One significant difference is, however, the fourth dimension in the General Relativity theory, which gives rise to a very different space structures and phenomena. What can one learn from the different types of experimental tests of General Relativity theory?

\subsection{Relativity Theory Is Sensitive for Specific Mechanisms, But less for Space Phenomena}

Among the successful predictions of General Relativity one could mention the deflection of light by the sun, the gravitational redshift of light, gravitational lensing, equivalence principle testing. These phenomena are just consequences of gravitation, including the equivalence principle and the absolute light velocity, introduced in the General Relativity theory as property of space. These mechanisms really exist, and therefore act. But for the success of experiments it is not clear whether they originate from empty space or from quantum processes.

General relativity is less sensitive with respect to predictions concerning space 
properties. It cannot tell whether the universe is static or dynamic. It cannot account for the inhomogeneous, granular appearance of the universe, since it treats it as homogeneous. It cannot say anything about the value of the Hubble constant nor about dark energy and dark matter, which are expected to occupy large areas in the universe. Singularities can be identified, but whether they are really Black Holes or just indicate where the theory fails remains an open question.

The author believes that this poor ability in dealing with space properties may in part be caused by the introduction of clock time into the fourth dimension of spacetime. It acts as a scale for measuring changes only, and, since it has no direct relation to matter or energy, cannot implement and communicate action.

However, in General Relativity tests, certain phenomena, attributed to gravitational distortion of space-time could be predicted and were tested: The perihelion precession of Mercury, in part attributed to spacetime distortions, can be calculated. For Mercury's perihelion movements by 575 arcsec/century, of which only 532 arcsec/century could be accounted for by classical Newtonian gravity calculations, General Relativity theory could explain the difference. Also deviations from geodetic precession (6 arcsec/year) and a Frame-Dragging Precession (0.039 arcsec/year) from Gravity Probe B Satellite experiments appear to support General Relativity and its four-dimensional space. But the effects observed are very small. The LIGO experimental setup, a Michelson-Interferometer for observation of gravitation waves, in 2015 detected a transient change of length of the order of one atomic diameter in form of half a dozen irregular maxima lasting together 2 tenth of a second. Are such tests a proof of space-time and gravitation waves or are other explanations imaginable?

The Dynamic Energy approach explains gravitation as information image of matter, aiming at decreasing and minimizing energy per state. It does that when interacting with matter and guides it like a remote-control system in an orbit subject to least action. This is different from the far-reaching action of Newton's gravitation and the near field action of gravitation in Relativity Theory. Already this is an interesting result, because remote control works technically and is commonly applied in steering drones.

When a travelling photon, particle and wave mediated via information on matter, is interacting with gravitation (information), there will be an effect of information acting on and changing due to additional information. There will be definitively an effect. This way deflection of light by heavy masses, the gravitational redshift, and gravitational lensing should in principle be explainable. It is also remarkable, that in form of remote control, using information (gravitation) on the spot to guide objects, irreversible nature applies a technology which our civilization has witnessed to be working. The open question remains, how natural objects can implement the provided information, when responding to gravitation. It should be recalled, that the Dynamic Energy theory is considering elementary particles as self-organized systems, comparable to virions, viruses de- 
coupled from energy supply and not characterizable as "living" organisms [4]. They are expected to "know" how to respond to natural laws.

The situation in challenging General Relativity is more complicated with phenomena interpreted as space-time properties. However, they are very small and there is also the possibility to find explanations on the basis of the energy driven Time Arrow approach. Here again gravitation fields are fields expressing and mediating information. Information has an energy content and energy can generate gravitation. Information fields can therefore interact with masses and can be distorted through their presence. This could account for some of the space related effects identified with General Relativity theory. A Frame-Dragging effect (Lense-Thirring effect), for example, should also be expected with an information (gravitation) cloud around a rotating mass. Significant efforts have been developed (especially via String-Theory and Supergravitation-Theory) to introduce quantization of gravity into General Relativity. They failed and Dynamic Energy theory can comment on that from its point of view: The function of information on matter during the dynamic particle-wave duality can be compared to that of a technical analogue-to-digital converter. Such a converter involves an algorithmic function which performs quantization of the analogue signal and is called a "quantizer". Information on matter, mediating the dynamic particle-wave duality (Figure 1 top right) may also act as such a quantizer, and by minimizing energy per state for an electron in an orbit of an atom or molecule it definitively can induce quantization of electronic states of atoms and molecules [1]. Within the Dynamic Energy approach the search for quantum gravity within General Relativity should be replaced by a profound study of information on matter. It plays a crucial role in quantum states and, as gravitation, is also decisive for understanding the universe.

\subsection{How to Deal with the Specific Experimental Evidence for Relativity Theory}

Because of the overwhelming experimental evidence claimed for Relativity Theory, criticism is not any more accepted by established journals. However, up to now critics could not present a reasonable alternative theory for explaining the always constant light velocity, gravitation, inertia, time behaviour and space properties. The Dynamic Energy model does this and claims in addition the potential of eliminating paradoxes and irrationalities of General Relativity. It also entirely naturally provides the link between quantum behaviour and cosmological function, and introduces, for the first time, the concept and mechanistic creativity of information technology into fundamental physical mechanisms. Our industrial civilization experiences the amazing potential of information technology, which is based on natural laws. Why should nature not apply them?

Here alternative explanations for experimental observations claimed to support Relativity Theory are sketched.

Time shifts of atomic clocks: Atomic clocks sent around the Earth or clocks in space show time dilation. It is interpreted to fully confirm Relativity Theory. 
These phenomena seen with atomic clocks in the sub-microsecond range occur with time intervals determined by atomic parameters only. According to the Time Arrow approach not the time changed, but a changed gravitation acting on the atomic clock modified the time lapses, determined by electronic transition in the atoms. Something similar would happen, if an ordinary pendulum clock would be taken up a mountain, where gravity, the acceleration imparted to objects, is lower. Its oscillation period inversely depends on the root of gravity and would become longer and correspondingly its oscillation frequency lower. This, however, does not mean that the time measured with the pendulum clock has changed. Just the scale used for measuring changes, the oscillations of the pendulum clock, experienced an alteration in the parameters controlling them. One would have to recalibrate the clock.

The Time Arrow approach explained quantization in atoms as consequence of minimization of information on matter [1] [6]. When additional gravitation (which itself also constitutes information on matter) is applied, the obtained minimum approached for quantization of electronic levels will be shifted and thus the distribution of the concerned electron orbits within the atom. This will change the time lapse during an electronic transition. Atomic clocks, today, can even register gravitation changes generated by the tides produced by the moon and react to a change of one meter in altitude. This has nothing to do with relativity theory. It cannot be claimed via experiments of travelling atomic clocks that time is relative, since the time lapses concerned are not the real dynamic time. What is observed is that a scale for measuring changes, the sequence of time lapses, which, when calibrated, we use as a clock, is altered by gravitation and additional parameters. Since such clocks are technologically important, this behaviour has, of course, to be technologically considered, but it does not support the claim of Relativity Theory that time is relative. It is also not a real space-time, but a "space-time scale", when just a scale for measuring changes is used to construct "space-time" and varies its intervals in dependence of gravity and other parameters. Relativity theory does not understand the real nature of time properly. It tries to understand the universe by linking it with a scale, lacking any relation to energetic processes and change, the properties of which are influenced by physical parameters.

\section{Diversion of light by gravitation}

Gravitation, being interpreted as information on matter, will have an effect on the trajectory of light, which is equally controlled and mediated by information on matter. A quantitative theory will be able to deal with this phenomenon, during which information enforces a minimisation of energy per state towards an implementation of the principle of least action in presence of additional information from outside. When gravitation (information) becomes strong enough, light will be visibly deviated and, in the case of a Black Hole environment, prevented from escaping. One is dealing with the effect of a "remote control" on elementary particles via information. 


\section{Increase of weight with increasing velocity}

The Dynamic Energy approach explains elementary particles and matter as self-organized energy [4]. Self-organized systems can grow in mass with energy from outside. Examples are a hurricane, a green plant growing in sunlight, a living species consuming food. The increase of mass during acceleration of elementary particles to very high velocities can be interpreted that way. Kinetic energy, when provided to a sufficient extent, is converted into mass. This is possible when mass is behaving as self-organized energy. The author believes, that Relativity Theory formally comes to the same conclusion, because of the physics introduced on the basis of its mathematical construction.

\section{The formula $E=m c^{2}$}

The famous relationship $E=m c^{2}$ derived from Einstein's theory of relativity describes the energy of a mass $m$ at zero velocity. It is considered synonym with Relativity Theory and any counter theory will have to deal with this situation. It is therefore important to learn that the derivation of this formula was not really based on logical considerations, but was already anticipated in the derivation of the result (see [16]). Classical deductions of analogue formulas were also described in the literature [17]. Einstein speculated that the energy formula he deduced for light, which included arbitrary assumptions, should also apply generally to any other energy form. Two years before Einstein, an Italian geologist, Olinto de Pretto, published the same relationship between energy and mass. He derived it from a non-relativistic consideration [18]. He observed how the mass of uranium and thorium transformed into energy during radioactive decay. Before him, around 1900, Henri Poincaré apparently brooded over the same formula. The point here is not to diminish the accomplishments of Albert Einstein. The point is that we want to understand what this formula actually means in the context of Relativity Theory. Do we need the Theory of Relativity to derive this proven formula? In reality, the formula $E=m c^{2}$ has nothing to do with relativity. It would still be valid if the four-dimensional space did not exist. Einstein had anyhow neglected relativistic considerations before deriving the famous energy-mass formula. This formula can be derived purely from classical arguments. The important energy-mass relationship can therefore not be used as a support and justification for a four-dimensional space-time. This is an important argument for the considerations here and justifies the discussion. One does not need the Theory of Relativity to obtain this important formula. And there is an additional relevant fact: The Dynamic Energy theory also conveniently establishes the connection between energy and mass. Mass is simply self-organized energy, it is consequently proportional to energy [4]. Self-organization is doing with energy what it does with water on a hotplate, when generating droplets erratically moving around (Leidenfrost-phenomenon). These water droplets made from water correspond to elementary particles generated from energy via self-organization. However, because of the complex mechanism of self-organization, the proportionality factor between energy and mass is ex- 
pected to be more complicated than $c^{2}$, which, in this simple form, accounts for the appropriate dimensions.

\section{Gravitation singularities are information singularities}

Gravitational singularities are locations within space-time, characterized by infinitely growing gravitation and undefined space-time properties. Dynamic Energy theory explains gravitation as information on matter with the task to decrease energy per state and thereby to reduce information contained in free energy. Such a developing singularity, called a Black Hole, is thus an extremely dynamic information phenomenon aiming at disrupting matter and generating entropy. During the proceeding mechanism information (gravitation) is increasingly concentrated. As explained in a preceding publication [2], this is a highly dynamic process, including self-organization and even structuring of information (gravitation). As a far from equilibrium self-organizing open energy consuming system it approaches maximum entropy generation, within given constraints, like derived for living systems [5]. The information driven matter consuming self-organizing Black Hole moves further and further away from equilibrium and finally develops quasar properties to get rid of accumulating entropy. One is dealing with an information controlled inorganic phenomenon comparable to primitive living organisms with a directional evolution and different development stages towards maximum entropy turnover.

\section{Gravitational waves as information pulses}

Within Dynamic Energy gravitation is explained as information on matter and information itself does not produce waves. But when self-organized, which is possible within the Dynamic Energy theory [6], time dependent, propagating information (gravitation) phenomena are to be expected. They have nothing to do with perturbations of a space-time structure of the universe and do not show a time-component of the assumed space-time perturbation (clock-time perturbations were not registered during the LIGO experiments). If space-time would exist, "gravitation waves" should be frequent, since the presently known universe contains 500 billion galaxies with over 100 billion stars in each. Space would be vibrating.

\section{Dark matter means self-organized information on matter}

Dark matter cannot be explained by the Theory of Relativity and when not found constitutes a problem for it. Dynamic Energy explains the dark matter phenomenon as self-organized information on matter (gravitation) [2] [6]. The time arrow allows feedback and thus a self-organization of information into a higher hierarchy of information handling with the task of reducing energy presence per state. The additional energy needed comes from the energy flow sustaining self-organization of information on matter. The result is much higher gravitation, powered by the energy consumed for the self-organization of information. Gravitation (information) could even get structured in space like observed in living organisms. No dark matter is needed and expected for explaining super-gravitation and gravitational lensing in the universe. The mirage effect 
is generated by self-organized gravitation (information) in a similar way as terrestrial mirage effects are generated be special, self-organized weather conditions.

\section{Space-time distortions versus dynamics of information clouds}

In the Dynamic Energy approach phenomena like the perihelion motion and curvature of space time could be reinterpreted as distortions and behaviour of information (gravitation) halos or information clouds around space objects. They have an energy content and may interact, for example, by responding to a rotating or otherwise moving mass. The behaviour of gravitation fields as fields of information and their mutual interaction need to be studied. How are information clouds behaving, which make up gravitation around masses?

\section{Cosmological redshifts versus information handled "tired light"}

Expanding radiation is, like an expanding gas subject to entropy production. The equivalence of the entropy formula applicable has already been used by Einstein in 1905 to justify the existence of light in form of particles (photons) [19]. His argument was that, subject to the same entropy formula upon expansion light should behave like particles of a gas. However, in time-neutral quantum physics photons, once released, are only allowed to lose energy by interacting with matter or gravitation. Entropy generation is not any more taken into consideration. Dynamic quantum physics requires and allows entropy generation of propagating photons via the mediating presence of information on matter. Since the logarithmic formula for entropy generation by expanding radiation into a larger and larger volume goes towards infinity, the energy of expanding, propagating photons should finally be consumed at the expense of released microwave radiation [2]. In contrast to abandoned classical theories on "tired light", which have already been discussed one century ago (Ritz, 1908 [20]; Zwicky 1929 [21]), light particles are not deviated by dust to produce a blurred sky, but get rid of microwave radiation without deviation from the photon path via the information on matter involved in energy redistribution. The redshift observed from distant stars and galaxies could therefore to a large extent be due to entropy generation by expanding, propagating photons and not to an explosive expansion of empty space (cosmological redshift) and accelerating escape movements of galaxies. On the basis of Dynamic Energy a new evaluation of structure and dynamics of the universe is required. It could be much more static than presently assumed.

\section{How accurate can bent space gravitation be measured?}

As scientific experience shows, a phenomenon such as gravitation is perfectly considered and implemented in natural processes. For this to work bent space gravity has to be registered with sufficient accuracy. Is this possible with a gravitation coined by a bent space, which has to be registered as an analogue signal? An indication of the accuracy in measuring gravity is provided by measurements (e. g. via torsion balances) of the gravity constant, which is deduced via the well-known formula relating gravitation forces to masses and their distance. Its presently recommended value is $6.67430 \times 10^{-11} \mathrm{~m}^{3} \mathrm{~kg}^{-1} \mathrm{~s}^{-2}$ with an uncertainty of $2.2 \times 10^{-5}$, which is mostly due to the fact, that the mass of the earth is not well 
known. This requires measurements in the laboratory with correspondingly quite small test masses and associated inaccuracies. The expectation prevails that an accuracy of 6 digits can be reached for the gravity constant. On the Earth surface the variations in gravity itself, which in average is $\mathrm{g}=9.81 \mathrm{~ms}^{-2}$, due to latitude amount to $+/-0.03$, due to local geological variations to $+/-0.0006$, and due to tides to $+/-0.000003$ [22]. The best accelerometers can measure gravity to two parts per billion, that is to $+/-0.000000002$ [23]. Such an accurate measurement can be performed on just the selected location. How can a bent space be measured so exactly without access to information on the degree and orientation of space bending?

\section{The role of self-organization}

Every self-organization requires a directed time which provides a "before" and an "after". A clock time does not provide that, because it only represents a scale, provided by oscillation phenomena, for measuring change. The Dynamic Energy approach, however, provides an energy driven time arrow which readily supports self-organization of matter and information (which has an energy content). The time orientation may be enforced by information loss accompanying energy processes from usable to unusable energy. Time is the loss of information on the past. This way, and because of the existence of a directed time, the complex space structures seen in the universe or in the structural complexity of life can readily be understood and explained. Such a creativity of the universe would not be possible within the time-neutral approach. Here daring mathematical procedures had to be applied to justify time orientation for self-organization (e. g. symmetry breaking). In practice, relevant disciplines, dealing with feedback and self-organization, e.g. control theory, presuppose a functioning of feedback processes, even though time neutrality and time as an illusion should not permit it.

The "Big Bang universe" of time-neutrality versus the "Self-Image universe" of irreversibility

The Big Bang universe (Figure 4, top) with the origin of energy from nothing, the inflation of empty space, with its quantum fluctuations, dark matter and the accelerating expansion of galaxies via dark energy is well known to be characterized by irrational assumptions (marked as $I_{1}$ to $I_{5}$ in Figure 4, top). However, practically full experimental verification is claimed [24] [25].

The Dynamic Energy approach for understanding the universe emphasizes the importance of information mediating between concentrated and distributed, chaotic energy. Such a mechanism applies for the particle-wave duality and is, since gravitation (information) dominates space, also expected to apply for the entire universe. A terminal, worn-out universe with a high entropy content will consequently be reconverted, by set aside information, into the free energy rich initial, original universe (Figure 4, bottom). We are dealing with a fractal universe, which shows parallel behaviour on quantum and cosmological level [2]. Such a behaviour is only to be expected from a fundamentally irreversible world, which is able to self-organize. Since the redshift of galactic light can, to a large 

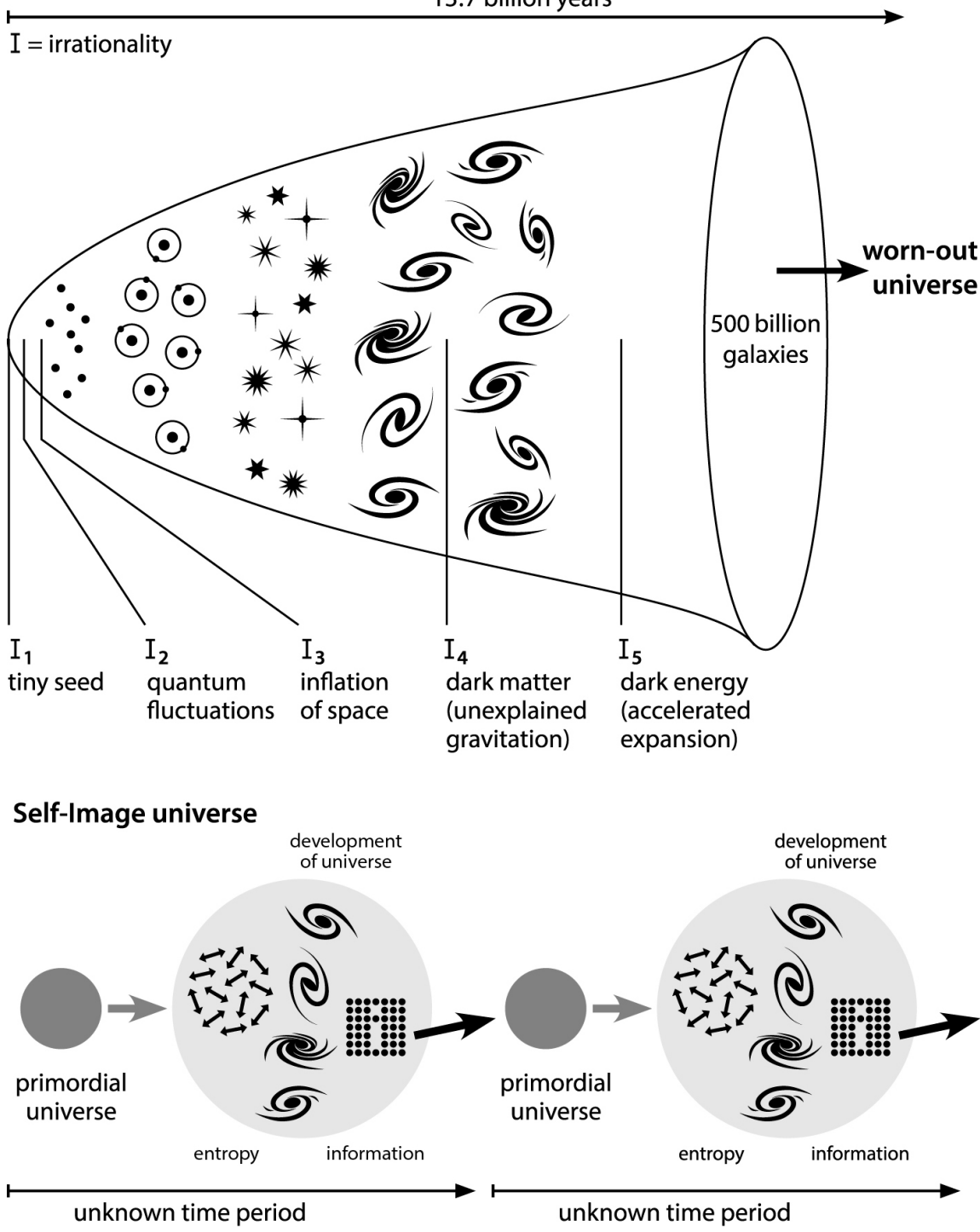

Figure 4. The Big bang scenario, which is sustained by the time-neutrality paradigm, shaped by General Relativity, and characterized by irrationalities (top) is compared with the Self-Image universe, resulting from Dynamic Energy considerations (bottom). The first describes an explosion of energy, matter and empty space without understandable sense, with life as chance phenomenon and an end in cold and darkness. The second starts via information, evolves galactic structures, live and spirit as an aim of self-organization, within an information-controlled universe, and regenerates itself finally again via set aside information.

extent, be understood as entropic energy losses (discussed above), most evidence for a Big Bang scenario can be interpreted differently and in support of the Self-Image universe [6].

But the Self-Image universe has additional striking properties. Because of its directionality (due to feedback-coupled mechanisms as shown for cybernetic systems [26]) and the role and importance of information it can explain evolution of consciousness and spirit. As matter can self-organize to living species, 
also information (related to energy and matter) can do this and reach a higher ranking or hierarchy and function. This occurs on the level of chemical information in relation to the genetic code and it also occurs in the brain, where upgraded, self-organized information leads to consciousness and spirit. Information on matter, gravitation, as well can self-organize under appropriate conditions and sufficient energy supply to yield super-gravitation, which can serve as a substitution for "Dark Matter", without the need for undiscovered dark matter particles (compare above). Feedback-coupled processes have teleologic character and follow an intrinsic aim [26], including in biological evolution, where genetic control is superposed to a strive of biological systems for maximum entropy production within their restraints [5]. The Self-Image universe is dominated by information (gravitation), and favours evolution of mind and spirit [6]. In contrast to the Big Bang universe without aim and with life just being a chance development, it is intelligent and creative, is logic and sophisticated [6], expecting further exploration.

\section{Discussion}

\subsection{A Credible Explanation of the Universe Is Simple and Rational}

For the first time, and based on reasonable starting assumptions (fundamental irreversibility in nature) a rational counter theory is proposed, which challenges the space-time understanding of the universe. Figure 1 explains, why the well-established time neutral world of space-time (Figure 1, left) has to be challenged by the energy driven Time Arrow world. The essential postulates by General Relativity theory, the always constant light velocity, gravitation and inertia as properties of empty space, can be explained differently, on quantum level and in a more straightforward way as consequences of a dynamically understood principle of least action. Information on matter, needed as a link between wave and particle aspect of matter, turned out to be a crucial aspect of fundamental irreversibility. Only one of the two theories, based on different paradigm, can be correct. If nature is fundamentally energy driven and irreversible, then a Big Bang scenario based on space-time concepts is wrong.

The "energy-driven Time Arrow" approach towards understanding a fundamentally irreversible universe, as an alternative to the established time-neutral one, which is shaped by General Relativity theory, is faced with a complex challenge. On one hand there is the claim that Relativity theory has passed every test. On the other hand the quest for fundamental irreversibility and an energy driven time has opened a very promising path: it eliminates paradoxes in quantum physics and has given straightforward explanations for gravitation (and inertia) and the absolute light velocity, without the need to introduce them as property of empty space. They are implemented on quantum level so that also the link with processes in the universe is automatically given. Gravitation turned out to be information on matter with the aim of decreasing the presence of energy per state. This yielded an explanation of gravitational forces in terms of a remote 
control of masses, distinct from Newton's long-distance action of gravitation forces and the close distance action of gravitation in General Relativity. Due to the orientation of the energy driven Time Arrow, a "before" and an "after" are readily given and consequently the route to self-organization is entirely open. It is open for energy, which self-organizes to elementary particles, for matter, which self-organizes to galaxies and life, and for information (which contains energy which is related to mass) which self-organizes to a higher hierarchy of information processing [27]. It yields a higher ranking of genetic information, when genetic information self-organizes. It is consciousness and spirit, when information in the brain self-organizes. It is super-gravitation when gravitation (information on matter) self-organizes. All together looks like a promising starting situation for the exploration of an intelligent universe.

When information on matter (gravitation) self-organizes in the inorganic environment of space, it moves up into a higher hierarchy. Then it also increases its order, or the information it contains. It may structure itself, with areas of high gravitation near others of low or no gravitation. The gravitational effect exerted becomes much stronger. The additional energy required for this supergravity comes from the self-organization of gravity, which demands a sustaining flow of energy. In any case, the supergravity proposed here, as self-organized information, does not require any dark matter. This dark matter has been searched for during four decades now. The complex experiments with the liquid Xenon probe in the Gran Sasso mountain in Italy were negative. The Chinese Panda-X-II experiments and the Swiss experiments with ultracold neutrons were also unsuccessful. The time arrow as a trace of energy does not need dark matter. Its effect is due to a dynamic self-organization property of gravitation, information about matter, which may regulate and dominate our universe in other respects as well.

Another thrust of the Dynamic Energy approach is access to irreversible thermodynamics of matter which the time arrow facilitates. The recognized limiting entropy law is "maximum entropy turnover within the constraints of the system" [5]. This applies for life (with the genetic mechanism superposed), as well as for galactic objects such as Black Holes [2]. In these it is information (explained to be gravitation), with the tendency to decrease energy per state, which increasingly concentrates and degrades matter. Because the products of entropy generation can, in an initial period, not leave the Black Hole due to gravitational attraction, its system is pushed further away from equilibrium. A new organization of the Black Hole and a structuring of gravitation is assumed which represents a Black Hole-Quasar association, which is able to exhibit maximum entropy (energy) turnover. The time arrow as a trace of energy implements this and is thus a key to the irreversibility and thus creativity of the universe. The Dynamic Energy universe explains observed phenomena rationally and in a straightforward way. Irreversibility and feedback mechanisms of self-organization are creative tools towards a structured and intelligent universe. 
However, time-neutral physics and the space-time world of General Relativity are well established [24] [25]. It is often stated that Special and General Relativity have passed every test. Some of these experiments were discussed, questioned and reinterpreted above. Since absolute light velocity and gravitation subject to equivalence with inertia are actually implemented in nature and were imposed on empty space via Einstein's field equations, some of the "verification" experiments may just reflect that. One verifies what the theory considered. But, as the "Dynamic Energy" approach shows, the ever-constant light velocity and gravitation could also have different origin. What is different, when light velocity and gravitation is controlled by information and originating from quantum processes? First, gravitation indeed occurs on elementary particle level. Towards a weight of one kilogram it increases by a factor of $10^{54}$. Second, important "demonstrations" of General Relativity have to be explained differently. As discussed above, the time dilation registered with travelling atomic clocks is not considered a change of time flow but simply to be the influence, which a change in gravitation is exerting on electronic states in the atoms, controlling the time lapses induced by electronic transitions in atomic clocks. It is well known that the Standard Model of elementary particles, which is based on time-neutrality, cannot explain gravitation at all. The "Dynamic Energy" approach, which considers matter as self-organized energy, can do it: particles exist and react as self-organized mechanisms. They grow and change during energy turnover like a hurricane. This permits also that accelerated particles increase their mass [4]. It explains also the diversity of elementary particles, of which only a few serve as useful building stones for matter. As discussed above, the equivalence of energy and mass is equally not a privilege of Relativity Theory. The Dynamic Energy theory has also the potential to provide alternative explanations for experimental results related to gravitation phenomena, interpreted as proofs for space-time. For this purpose more has to be learned about the dynamics of information (gravitation) clouds.

\subsection{The Time Arrow Universe Is Rational, Intelligent and More Fascinating}

The Self-Image universe drawn by the Dynamic Energy theory is recreated from a worn-out universe by set aside information on matter and can develop successive activity periods (Figure 4, bottom). The energy driven Time Arrow leads to a self-organization of energy to elementary particles [4]. Among these, which were compared with virions, viruses without access to energy, some are useful as building stones of matter. Also matter can self-organize and thereby already uses self-organized genetic information to upgrade its creative and sustaining abilities. Structured, self-organized matter aims at maximum entropy turnover and thereby prepared the conditions to support self-organization of information in the brain. The brain requires a high rate of energy turnover for self-organization. Consciousness and spirit evolved [5]. Evolution of spirit within an information-based universe turned out to be the aim of evolution. Its origin is purely 
materialistic and rooted in the feedback-controlled nature of self-organization processes [26]. All together the contours of a spiritual universe appear, which sees evolution and life not as a coincidence, but as part of a gigantic experiment triggered by energy acting via the time arrow. Far reaching philosophical considerations result. Also the question can be answered, what nature actually is. It is a question which could not be answered on a purely materialistic basis before. The Time Arrow can now do it:

"Nature is the self-realization of energy over the time arrow".

Due to its fundamental irreversibility, energy can self-organize to elementary particles and to matter [4]. Matter can self-organize to structured objects and life [5]. Information in structured matter can self-organize to generate mind and spirit [6]. The time arrow, of course, is action time, the flow of energy driven action, equivalent to the loss of information on the past. Relevant questions around such conclusions and arguments leading to them are discussed in a recent monograph [27].

The paradigm of time neutrality and Relativity Theory has, in contrast, sketched a universe which starts with energy from nothing in a Big Bang explosion. It involves a (bizarre) expansion of empty space and recognizes an evolution, which functions by pure chance and natural selection. This concept of evolution is characterized by no aim, cannot explain its obvious thrust and is unable to explain consciousness and spirit. The chaotically starting and dramatically expanding universe consumes its energy resources and is finally heading towards a cold death in infinite expansion. Mostly puzzling is the fact, that such a highly dynamic universe was constructed starting from assumed time-neutral particles and natural laws. Critically seen it does not explain the dynamics it created. Such a concept does not allow relevant philosophical questions either.

The new vision of the universe, the Self-Image universe (Figure 4, bottom), which is started, largely controlled, and regenerated by information, is fundamentally irreversible, explains dynamic change, aim and creativity, and interestingly shows rationally understandable contours of development and destination. It is profoundly logic and philosophically highly attractive, because questions can be asked with respect to the role, development and aim of spirit, which is part of its character [27].

\subsection{Time Neutrality versus Fundamental Irreversibility}

The Dynamic Energy approach claims that the Time-Neutrality paradigm, applied to highly dynamic processes, is largely responsible for relevant paradoxes and finally also was responsible for the rise of Relativity Theory based on clock time, which has no relation to energetic processes. How the basic assumptions of the time-neutrality paradigm led to well-known, presently still tolerated, paradoxes is analysed in Figure 5.

Time-neutrality and an energy which is just a scalar without any relation to change is seen as the reason why the dynamic nature of the principle of least 


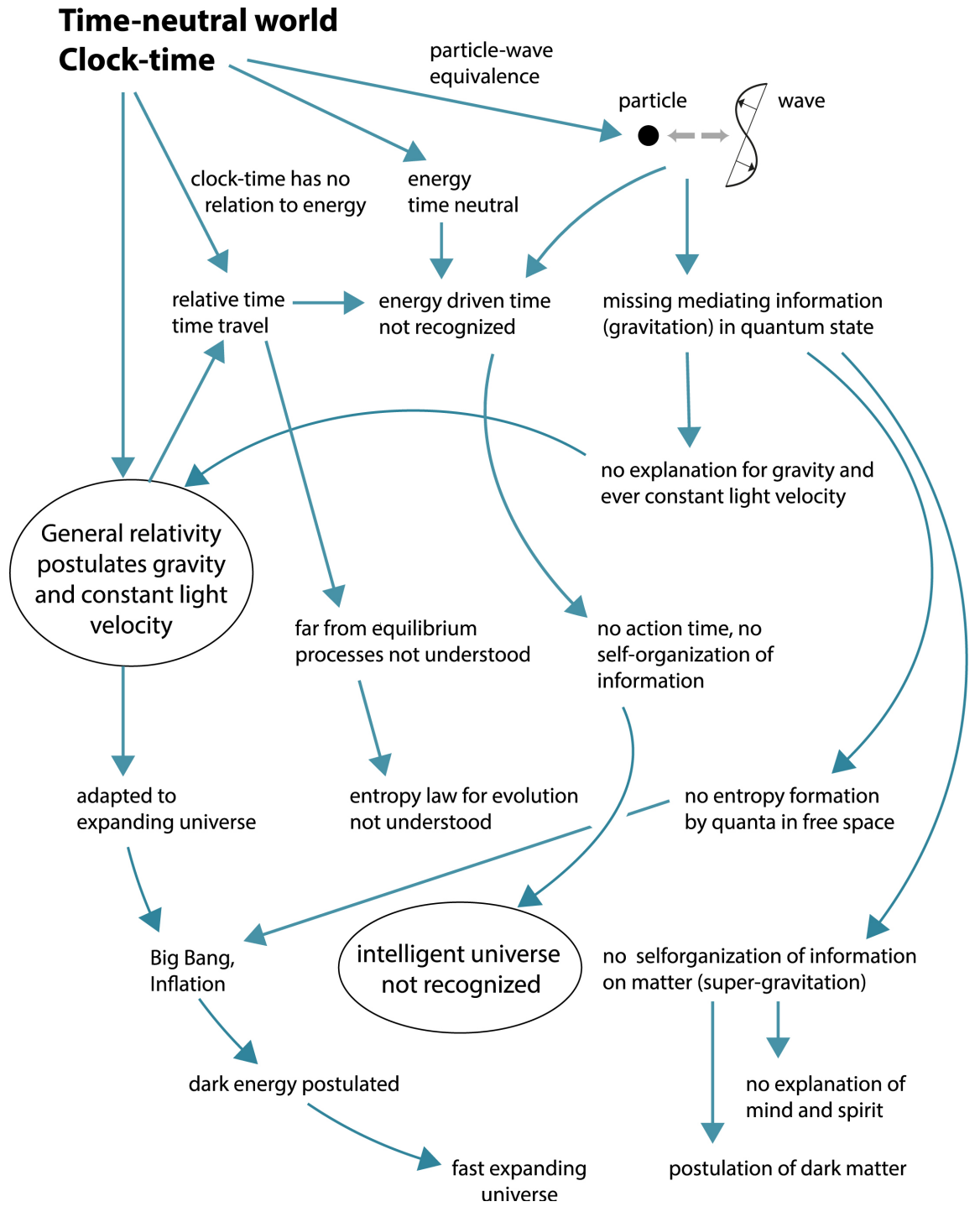

Figure 5. Explains how the assumptions of a time-neutral nature and the classical particle-wave duality have led to paradoxes and irrationalities in historically grown science and justified the rise of Relativity Theory. Assuming a Dynamic Energy derivable from a dynamic interpretation of the principle of least action, and thus a fundamentally irreversible nature, eliminates paradoxes and explains a very different universe (Figure 1, right).

action and thus fundamental irreversibility was not understood [1]. A consequence also was the assumed energy-equivalence of particle and wave in quantum theory with the resulting well known quantum paradoxes. The necessity to consider information on matter as mediator in the particle wave duality (which avoids paradoxes) was not recognized either. Because no explanation for the ever-constant light velocity and for gravitation was therefore evident this led to the astonishing claim of Relativity Theory that empty space generates these phenomena. In empty space spreading and propagating photons cannot, within standard quantum theory, generate entropy. Photons would have to interact with matter or gravity. As a compensation, inflation and expansion of empty space 
has been assumed in support of the Big Bang scenario.

The energy driven Time Arrow approach thus also provides, in Figure 5, explanation for the cause and development of paradoxes and irrationality within the time-neutral space-time concept. This is an additional argument for the reality of a fundamentally irreversible world and the need for a paradigm change.

The Dynamic Energy theory which can be derived from a dynamic interpretation of the principle of least action [1] applies just the additional condition for quantum physics that energy, diluted in time ad space, has less ability for work compared to concentrated energy. Starting from the paradigm change of dynamic energy and respecting the role of space and time for energy appears to be sufficient for explaining essential quantum and cosmological phenomena and arriving also at a reasonable philosophical interpretation of evolution and the universe (Figure 1, right side).

The above given alternative interpretations of experiments, that successfully seem to support Relativity Theory, show that they do not contradict a fundamentally irreversible nature. Alternative, and in addition logic and simpler interpretations in line with Dynamic Energy theory are possible. No experiment has up to now been communicated that unambiguously shows that a natural phenomenon can be inverted in time without additional changes in the environment. Such efforts should be continued as an attempt to support or challenge Relativity Theory based on the time-neutrality paradigm. Also, answers should be found to questions on relativity as sketched in Figure 3. The author insists that there should be no tolerance for irrational concepts in science and the step from clock-time to an energy driven action time (Figure 2) would quite radically change understanding in cosmology.

In support of such a step it should be explained how measurement values for gravitation covering 54 orders of magnitude (between two neutrons and two one kilogram weights) can be expressed and registered in form of a bent space around spherical material objects. For the expected analogue signals expressing gravitation based on a bent space this appears to be impossible. Only 4 orders of magnitude $(0.01 \%$ of full value) can reliably be measured with such systems. On the other hand, gravity measurements with an accuracy of 2 parts per billion have been achieved, as explained above (compare [23]). Besides of the LIGO experiment the gravity probe $B$ experimental results provide another example of surprising accuracies while dealing with quite small signals. The already mentioned measured frame dragging drift around rotating Earth, which was claimed to be in good agreement with General Relativity, amounted to approximately $10^{-5}$ parts of one degree per year [28].

Can the bent space of General Relativity around Earth be registered within an accuracy of 5 to 9 digits (decimal positions)? In order to do that the instrument (or the physical object concerned) must be able to retrieve or register the corresponding information from the curved structure of space, which is expected to 
be present and active during measurement (or interaction). The discussed measurable gravity values already indicate a two to six orders of magnitude higher accuracy than expected for a measurement of an analogue signal which a bent space is able to provide on the basis of practical experience: the error of an analogue instrument due to gain is generally estimated to be $0.01 \%$ or three digits behind the comma, to which an offset error has to be added (e.g. [29]). And there is an additional problem: bent interfaces or space regions as measure for gravitation cannot be properly evaluated by just measuring one point. A particle arriving and selecting a curved trajectory around a spherical object has to monitor curved space gravitation. Several or many points apart are needed to approach such a bent trajectory or space or one needs to scan it for digitalization and evaluation. This is evident, since the degree of bending is a measure for the intensity of gravity. Can a bent space reflecting gravity, which undoubtedly provides an analogue signal, really be measured with such an accuracy, and at one location only, as explained above? How can a gravity measurement of bent space on just one location yield an accuracy of 9 digits [23]? The author's conclusion is that this is not possible with the space-time gravitation mechanism of General Relativity. This is a strong, experimentally verifiable argument against bent space gravity. In spite of many contrary claims, and an elaborate created knowledge basis on Relativity Theory [30] [31] [32] [33] [34] such a concept of space-time and gravity does not match reality.

With a gravitation in form of information in numbers, on the other hand, registering and handling such a signal over many orders of magnitude, and providing such a sensitivity on any single location where gravitation exists would work. Measurement of gravitation on one location only is sufficient for obtaining the necessary information, as actually possible in reality. The measurement is then dealing with an information cloud around matter, in which information on matter, gravity, is present, properly distributed and available and active in form of numerical data. Can information better explain gravity? In a given gravitational field, all bodies, whether light as a feather or heavy as a hammer, are subjected to the same acceleration. They approach the ground at the same speed, provided that no air is present to exert varying amounts of friction. Such an experiment was actually carried out successfully in 1971 by Apollo 15 astronaut David Scott on the moon. One can rationally understand that the information image imposes such a behaviour, an equally strong acceleration, on masses. The information given in an identical gravitational field for a reduction of energy per state is simply the same for differently shaped objects. So also the observed acceleration is identical for a feather and a hammer. The principle, according to which acceleration does not depend on mass, shape or density of an object, is thus comprehensible, logically understandable. It is triggered by the same implemented information. However, the force experienced by unequal objects is different, since the triggered acceleration must be multiplied by the corresponding mass. 
How nature is actually handling information on matter in the form of gravitation needs, of course, to be explored. It may be a demanding task, but a realistic one, compared to the claim of Relativity Theory that curving of empty space is doing that. Elementary particles are already showing gravity properties so that the phenomenon must originate in them as derived by Dynamic Energy considerations. One knows what to search for, and what questions to ask. Experience within our evolving information age may provide more and more technical clues.

Such a measurement challenge, the distinction between analogue space-time signals on gravity and digital Dynamic Energy signals of gravity is proposed here as a falsification criterion (according to Popper [35]) for Relativity theory and Dynamic Energy theory respectively. Dynamic Energy involves information on matter in form of clouds around masses as explanation for gravity, in conflict with General Relativity with its bent space around masses. Since information on matter, as gravitation, turned out to be so essential as the link between quantum physics and cosmology, and as a key to a more intelligent Self-Image universe (Figure 4), another falsification effort should concern this information: It should be attempted to demonstrate that for a mutual transformation between two different material phenomena such as particle and wave no mediating information would be needed. The author has pointed to the example of analogue-digital-analogue converters in cell phones as functioning technical devices for digitalizing, evaluation and reconstruction [27], which show that natural laws require a mediating information program for a working exchange between wave and particle (compare Figure 1, top right). Omitting this information (as occurring in classical quantum theory on the basis of time-neutrality) prevents dynamic function and must lead to problems and contradictions in understanding. The Dynamic Energy approach requires this mediating information, which also turned out to be crucial for eliminating quantum paradoxes and for building a rational link to cosmology. Falsification is successful, if it can be demonstrated that the mediating information between particle and wave (dotted squares in Figure 1, right side) is not needed.

The Franciscan monk William of Ockham proposed in the 14th century that "no more causes for natural events should be allowed than absolutely necessary for their explanation". This rule of thumb for scientists, also known as Ockham's razor, would clearly favour the Dynamic Energy approach claiming a fundamentally irreversible nature, presented here, over the irrational theories, including General Relativity theory, for various natural phenomena, based on time neutrality and criticised in this paper. Figure 1 shows that much less and more reasonable starting assumptions (marked with rectangles) are required (right) compared to the time-neutral space-time approach (left). In addition, irreversibility is not a claim but results from a dynamic interpretation of the principle of least action [1]. Within the paradigm of an energy-driven Time Arrow historically evolved time-neutral theories that lead to irrational conclusions could be 
challenged, adapted and replaced, and this with significantly more reasonable basic assumptions [6] [27]. Moreover, it is not the same to describe nature through paradoxes and to declare them fundamental as to explain nature rationally as the Dynamic Energy approach can. Common sense would always prefer rational explanations and consider them simpler and making more sense. Last not least the fundamentally irreversible nature, as derivable from a dynamic interpretation of the principle of least action, explains evolution of spirit in context with a more conciliatory and supporting universe, which is rational and in principle much simpler than the Big Bang scenario. Relativity Theory would not allow that, because it relies on time-neutral particles and laws, claims a complex space-time, uses a clock which does not reflect, but only monitors change, claims empty space as origin for the always constant light velocity as well as for gravitation and inertia, speculates with an exploding vacuum and more recently even with multi-worlds.

Dynamic Energy pictures a much simpler, rational and more attractive universe. It is a promising alternative, because truth regularity proved to be simpler and philosophically more rewarding. It especially promises to allow penetrating deeper into natural contexts. There will never be a reasonable scientific understanding of bent empty space gravity of Relativity Theory, but the information technology expected behind the information-based gravity of the Time arrow approach promises a deep penetration into the secrets of the universe. The proposed new truth is that nature is fundamentally irreversible, that real time flow is the loss of information on the past, that the two Relativity Theories design a fictional universe by assuming that gravity and always constant light velocity are properties of empty space. Existing nature is, in principle, much simpler than presently seen and it is rational, while able to creatively evolve sophisticated structured systems including galactic objects, life and spirit within a universe dominated by information.

\section{Conflicts of Interest}

The author declares no conflicts of interest regarding the publication of this paper.

\section{References}

[1] Tributsch, H. (2016) Journal of Modern Physics, 7, 365-374. https://doi.org/10.4236/jmp.2016.74037

[2] Tributsch, H. (2016) Journal of Modern Physics, 7, 1455-1482. https://doi.org/10.4236/jmp.2016.712133

[3] Susskind, L. (2012) Modern Physics, General Theory of Relativity, Fall 2012, Stanford.

https://podcasts.apple.com/us/podcast/modern-physics-general-theory-of-relativity -fall-2012/id571368922

[4] Tributsch, H. (2018) Journal of Modern Physics, 9, 1361-1380. https://doi.org/10.4236/jmp.2018.97082 
[5] Tributsch, H. (2018) Advances in Anthropology, 8, 140-174. https://doi.org/10.4236/aa.2018.83008

[6] Tributsch, H. (2015) Irrationality in Nature or in Science? Probing a Rational Energy and Mind World. CreateSpace.

[7] Pais, A. (1979) Reviews of Modern Physics, 51, 863-914. https://doi.org/10.1103/RevModPhys.51.863

[8] http://www.kritik-relativitaetstheorie.de http://www.anti-relativity.com

[9] Climont, J. (2012) The Worldwide List of Dissident Scientist. https://de.scribd.com/doc/229428780/The-Worlwide-List-of-Dissident-Scientists-Je an-de-Climont

[10] Arteha, S.N. (2003) Criticism of the Foundations of the Relativity Theory. http://vixra.org/pdf/1201.0082v1.pdf

[11] Tributsch, H. (2008) Energie, Zeit und Bewusstsein. Shaker Media, Aachen.

[12] Withrow, G.J. (1980) The Natural Philosophy of Time. Clarendon Press, Oxford.

[13] Zeh, H.D. (1992) The Physical Basis oft the Direction of Time. Springer, Berlin. https://doi.org/10.1007/978-3-662-02759-2

[14] Reichenbach, H. (1956) The Direction of Time. University of California Press, Berkeley. https://doi.org/10.1063/1.3059791

[15] Davies, P.C.W. (1974) The Physics of Time Asymmetry. Surrey University Press, Guildford.

[16] Ives, H.E. (1952) Journal of the Optical Society of America, 42, 540-543. https://doi.org/10.1364/JOSA.42.000540

[17] Gut, B.J. (1981) Immanent-logische Kritik der Relativitätstheorie. Oberwil b. Zug: Kugler, 151S.

[18] Olinto De Pretto (1903). http://en.wikipedia.org/wiki/Olinto_De_Pretto

[19] Einstein, A. (1905) Annalen der Physik, 17, 132. https://doi.org/10.1002/andp.19053220607

[20] Ritz, W. (1908) Annales de Chimie et de Physique, 8, 145.

[21] Zwicky, F. (1929) Proceedings of the National Academy of Sciences, 15, 773-779. https://doi.org/10.1073/pnas.15.10.773

[22] https://web.calpoly.edu/ gthorncr/ME302/documents/AccuracyofGravity.pdf

[23] https://en.wikipedia.org/wiki/Gravimetry

[24] Will, C.M. (2006) Living Reviews in Relativity, 9, 3. https://doi.org/10.12942/lrr-2006-3

[25] Fließbach, T. (2003) Allgemeine Relativitätstheorie. 4. Auflage. Elsevier-Spektrum Akademischer Verlag, Amsterdam.

[26] Rosenblueth, A., Wiener, N. and Bigelow, J. (1943) Philosophy of Science, 10, 18-24. https://doi.org/10.1086/286788

[27] Tributsch, H. (2019) Time Arrow as Trace of Energy. Logical Key to a Spiritual Universe, MyMorawa, Vienna.

[28] Gravity Probe B. https://en.wikipedia.org/wiki/Gravity_Probe_B

[29] https://www.mccdaq.com/TechTips/TechTip-1.aspx

[30] Compère, G. (2019) Advanced Lectures on General Relativity. Springer, Berlin. https://doi.org/10.1007/978-3-030-04260-8 
[31] Dick, R. (2019) Special and General Relativity: An Introduction to Spacetime and Gravitation. IOP Publishing, Bristol. https://doi.org/10.1088/2053-2571/aaf173ch1

[32] Guidry, M. (2019) Modern Relativity Theory, Black Holes, Gravitational Waves and Cosmology. Cambridge University Press, Cambridge.

[33] Deruelle, N. and Uzan, J.-P. (2018) Relativity in Modern Physics. Oxford Graduate Texts, Oxford. https://doi.org/10.1093/oso/9780198786399.001.0001

[34] Luscombe, J.H. (2018) Core Principles of Special and General Relativity. CRC Press, Boca Raton. https://doi.org/10.1201/9780429023835

[35] Popper, K.R. (1979) Die beiden Grundprobleme der Erkenntnistheorie. J.C.B. Mohr (Paul Siebeck), Tübingen, 426-427. 